\title{
Analysis of domestic and overseas carbon trading market
}

\author{
Jianfang Zong*, Liang Chen, Liang Sun, Huiting Guo
}

China National Institute of Standardization, No.4 Zhichun Road, Haidian District, Beijing, 100084

\begin{abstract}
With the rapid development of international carbon finance, the carbon finance market has already been developed worldwide, including China, and has a very good development potential in the future. This paper first introduces the background of carbon trading, analyzes the domestic and overseas carbon trading market, and studies the construction framework of China's carbon trading market. This paper also elaborates the analysis of global carbon trading market development status. Finally, the analysis of China's carbon trading countermeasures are provided as per the aforesaid analysis results.
\end{abstract}

\section{INTRODUCTION TO CARBON TRADING BACKGROUND}

The carbon trading is the market mechanism which is adopted to promote the global greenhouse gas (GHG) emission reduction and reduce the global carbon dioxide emissions. The Intergovernmental Panel on Climate Change (IPCC) passed the United Nations Framework Convention on Climate Change (UNFCCC) on May 9th, 1992. In December, 1997, the first supplemental agreement of UNFCCC, namely, Kyoto Protocol (hereinafter referred to as Protocol), was adopted in Kyoto, Japan. As per the Protocol, the market mechanism is used as a new path to solve the carbon dioxide-dominated GHG emission reduction problem, namely, the carbon dioxide emission right is deemed as a commodity, which further forms the trading of carbon dioxide emission right, also called carbon trading for short. In the light of the types of GHG emission reduction, emission reduction timetable, and verified emission reduction, and so on of the major developed countries, Protocol makes the specific regulations, determines three types of emission reduction mechanism, namely, Joint Implementation (JI), Clean Development Mechanism (CDM), and Emission Trading (EI). Protocol lays a foundation for the development of the carbon trading market. This Protocol has come into effect since February 16th, 2015, and 184 countries have signed this Protocol as of 2010. Among these three mechanisms, only the CDM involves the developing countries. Therefore, carbon resources are deemed as strategic resources, while the better and faster development of carbon trading must gain the increasing attention from people in all walks of life. There are also many carbon exchanges listed all over the world, for example, EU, Australia, New Zealand, and etc [1].

\section{ANALYSIS OF OVERSEAS CARBON TRADING MARKET}

Since Kyoto Protocol went into effect, the global carbon trading market has been formed after nearly ten years of development, and gradually become the significant power to promote the development of low carbon economy. EU, USA, and other developed countries have already established the relatively perfect carbon market, setting themselves ahead of the developing countries. The analysis of overseas carbon market is shown as below:

\subsection{The market segmentation degree has high level}

There are many standards for the classification of existing international carbon trading market. First, it will be classified according to the source of carbon emission right, the carbon market may be divided into the allowance-based market and project-based market. The allowance-based market is subject to the allowance allocated by the administrator, while the participants will buy and sell the emission allowance as per the demands. The allowance-based market may be further divided into the compulsory carbon market and voluntary carbon market. On the project-based market, the project with the emissions below the baseline level or the carbon sequestration project, after being verified, may receive the Emission Reduction Unit (ERU), for instance, the ERU generated from JI project, and Certified Emission Reduction (CERs) generated from the CDM projects. Second, it may be divided by the region into the European Union Emission Trading Scheme (EU-ETS), North American Emission Trading Scheme, Oceania Emission Trading Scheme, and Asia Emission Trading Scheme [2].

\footnotetext{
* Corresponding author: zongjf@cnis.gov.cn
} 


\subsection{The trading mechanism is relatively perfect}

The international carbon market begins early, while the allowance-based and project-based trading mechanisms have already approached perfection. Besides, the international carbon market trading can also be combined with these three auxiliary trading mechanisms of international cooperation emission reduction mechanism, namely, Joint Implementation (JI), Clean Development Mechanism (CDM), and Emission Trading (EI). These three mechanisms can facilitate the realization of international cooperation of mitigation climate change, and it is also conducive to the effectiveness of clost allocation in achieving global climate change, and to achieve a win-win situation between both countries in the trading.

Generally, the present international carbon trading market is dominated by allowance-based market, followed by the project-based market. The trading system of western developed countries has a much longer and wider practice in trading mechanism, so their development has entered the mature stage.

\subsection{The market regulation is perfect}

Whether the market regulation is perfect or not is a significant standard to measure whether a market is mature. On the international carbon market, some trading systems establish their own regulation mechanism. In which, EU's regulator of carbon trading involves the European Commission and regulator of EU member states. In each member state, the regulator of carbon market is its own environmental protection authority. The American carbon trading regulator does not have an overall responsible organization. Instead, the state regulator is in charge of governmental regulation, while the regional regulator without the administrative subject qualification, is in charge of the administration and technical support.

\subsection{The financial institutions shall actively participate}

Not only the carbon emission right itself can be traded, but also its derivative financial products can have the extensive trading on the overseas market. The financial institution shall extensively participate in every link of carbon trading. For instance, the CDM-based secondary market participating in the CERs (Certified Emission Reduction) spot, forward and future trading develops very rapidly. The involvement of financial institution, can not only help the enterprises and governments to avoid the risks resulted from the uncertainty of emission reduction projects, but also gain the profit from a new perspective to realize the win-win of economic and social benefits.

\section{CURRENT STATUS OF CHINA'S CARBON TRADING MARKET}

On December 19th, 2017, China's carbon trading market was officially initiated, and the trading system construction is accelerated. At present, many types of carbon trading market have already been established worldwide. China has carried out the carbon emission right trading pilot respectively in Beijing, Shanghai, Chongqing, Hubei, Guangdong, Shenzhen and other seven provinces and cities nationwide, and completely initiate the online trading. By the end of 2017, the accumulated trading volume in seven pilot carbon markets has already broken through 200 Million tons, while the accumulated trading amount has already exceeded 4.7 Billion RMB.

In general, the market performance of each pilot carbon market greatly varies, which is correlated with the difference of local energy consumption, carbon emissions, economic development level, and governmental regulatory effect. Since the market was open, the average trading price of each China's pilot carbon market is about RMB 20 per ton, which is still much lower than the estimated marginal abatement cost. The carbon quotation trading price on the pilot market still cannot fully and effectively reflect the carbon marginal abatement cost in the thermal power industry. On December 19th, 2017, after being agreed by the State Council, National Development and Reform Commission (NDRC) issued the "Program for the establishment of a national carbon emissions trading market(Power generation industry", which symbolizes that China's carbon emissions trading system has already completed the overall design, and been officially initiated. Using the power generation industry as a breakthrough, it is intended to steadily promote the carbon trading market construction works in three phases, namely, basic construction phase, simulation operation phase, and deepening phase.

In order to accelerate the construction of carbon trading market administrative system, the national competent department will accelerate and promote the introduction of interim regulations on the administration of national carbon emissions trading, study and develop the administrative measures on the enterprise emissions report, market trading administrative measures, and other significant complement management system. Simultaneously, the quotation allocation work is also preceded orderly. Among which, in power generation industry, in addition to the power generation enterprises, electric network line loss will also be included into the carbon trading market management scope. According to the scheme regulations, each industry shall adopt the "Industrial benchmark methodology" or "Historical emission intensity reduction methodology" to allocate the allowance.

In order to enforce the "Program for the establishment of a national carbon emissions trading market(Power generation industry)", the work programs are successively proposed nationwide. For instance, the Sichuan province proposes to develop and improve the carbon market management system and participate in promoting the construction of carbon market support system, and carry out the carbon market monitoring, report and verification, strengthening the capacity 
construction of carbon market participants, actively cultivating the carbon market related extended industry, and other major tasks. At present, the construction of national carbon emission right registration system and trading system is carrying out the preliminary works, while the 2016-2017 historic data report, accounting, and verification, and relevant preparation works are also conducted accroding to the relevant national requirements nationwide. Besides, the competent authority will also strengthen the intensity of market capacity construction and management works upon each department, key emission units and third-party verification institutions, and etc.

The ten thousand tons of standard coal entry threshold enables the national carbon market to cover almost all the major thermal power generation enterprises. From 2018 to 2019, the national carbon trading market construction will be at the basic construction and simulation operation phase, which temporarily will not cause the actual influences upon the power generation cost in the power generation sector. It is expected that only after the national carbon market initiates the allowance spot trading in 2020, the carbon trading will gradually come into play in controlling the carbon dioxide emissions and reducing the carbon abatement cost in the power generation section.

The fusion of carbon emission right trading system and other energy and climate policies, will be the key to the next steps. If the current electricity price control mechanism remains the same, it is hard to establish the effective carbon pricing affected transmission mechanism and deliver the price signal to the electric consumers, which will finally damage the original design intention of promoting the industrial carbon abatement by means of the market method. When the time is ripe, it is proposed to use the carbon tax as the supplementary means to realize the aforesaid carbon abatement by means of the market method. It is suggested to summarize the domestic pilot achievements, draw useful experiences from foreign countries, and further strengthen the top level design, making the carbon emission control target dominate the policies of energy conservation, renewable energy sources and other relevant fields, and avoiding the inharmonious and even disputed policies among different fields.

At present, China's carbon market is still in the early stage, and is lack of the advisory, research and broking services provided by the broker professionally engaged in carbon emission trading, which causes that we cannot conduct the effective communication and negotiation with the country of buyer and the relevant parties of UN. Besides, due to the inconsistency between the domestic and international standards, a large number of domestically accepted CDM projects cannot receive the accreditation from the UN CDM Executive Board. In the light of China's current fundamental realities and its own funds, technology, and capacity building restrictions, China is still a CDM market of great potential, because China is not only one of the top GHG emitters, but the biggest possible victim of greenhouse effect. The development of carbon market becomes one of the most important issues at present. However, due to China's later start in carbon trading development, and the restrictions of China's actual realities nationwide, China's carbon trading market development still has many problems as follows: the defective system, weak monitoring and regulation of carbon emission source, seriously fragmented trading markets, lack of the definite rewards and punishment mechanism and fairness, inadequate knowledge about China's carbon trading, the great potential policy risks, and lack of the professional talent support, the loophole existing in the carbon finance trading and operation system which makes it prone to the influence of market environment.

\section{RESEARCH ON THE CONSTRUCTION OF CHINA'S CARBON TRADING MARKET FRAMEWORK}

China's macro policy and legal environment regarding energy conservation and emission reduction has already taken shape, while the perfect and stable carbon trading market is the significant premise that can ensure the carbon trading practice to be normally proceeded. Research results regarding the construction of China's carbon market framework are mainly demonstrated as follows:

\subsection{The fundamental target of the carbon market framework construction}

The purpose of the carbon market framework construction is to achieve the ideal emission reduction effect and sustainable development of ecological environment with the minimum economic and environmental cost, and the assistance of the adjustment function of market mechanism, and also optimize all types of energy conservation and emission reduction resources, instead of only constructing the carbon market. In other words, the construction of China's carbon market framework must follow and combine the national economic growth, to properly arrange the carbon emissions, and promote the balance between carbon emission right trading price and emission cost. Generally, China's carbon market framework is the trans-regional or national trading market dominated by the compulsory market and followed by the voluntary market [3].

\subsection{The construction pattern of carbon market framework}

In view of the construction conditions of the carbon market of every country, there are three types of construction patterns of carbon market, namely, topdown pattern, bottom-up pattern, and mixed pattern. The top-down pattern is more suitable to countries that have a certain carbon market foundation, which is intended to construct the carbon market by mainly developing the carbon market laws, policies and operation mechanism, which covers the whole country and region. This type of construction pattern is the most efficient method to construct the carbon market framework. However, such 
pattern cannot properly satisfy the inner demand of the participation enterprises. The bottom-up pattern is more suitable to the countries where the local enterprises have a much higher demand in carbon emission trading, which will officially initiate the construction works after the construction scheme, proposed by mainly drawing successful experiences of regional carbon market construction from other countries. It is reviewed and approved by the relevant department of the government. If the market framework is constructed by using such pattern, it will lay a solid foundation for the national carbon trading market. However, such pattern is more suitable for the construction of regional carbon trading market framework. Once the regional carbon market takes the shape, it is likely to impede the construction of unified carbon trading market nationwide. The mixed pattern is intended to consciously and actively construct the regional carbon market in combination with the actual demand of local development. When the regional carbon market has been developed maturely and steadily, the state will collectively unite all the regional carbon markets to form a national carbon market. Doubtlessly, mixed pattern covers the advantages of the other two patterns. However, such pattern also has the disadvantages, namely, it may face many difficulties when uniting all the regional carbon markets. In light of China's fundamental realities and carbon trading demands, it is most suitable to construct the carbon market with the mixed pattern.

\subsection{The core mechanism of carbon trading market operation}

The construction framework of China's carbon market related mechanism mainly covers three significant modules, namely, improving the carbon emission trading related legislation, determining the carbon trading operation mechanism, and strengthening the development of regional carbon market connection mechanism. The improvement of existing carbon emission trading related legislation contributes to the complete achievement of legal binding force of national carbon emission right, which is the major strategy to ensure the carbon trading activity to be proceeded normally, and the carbon market order is stable. Therefore, in the future, China shall accelerate the legislation process of carbon emission right trading related laws and regulations. The development of "Carbon emission trading law of the People's Republic of China, has a definite division upon all aspects of carbon emission trading activities, and also provides the definite punishment system upon the violation acts against the relevant laws and regulations. Besides, it shall release the matching laws and regulations, explicitly defining the major manners and specific procedures of China's carbon trading activities. It is intended to adopt such a series of measures to further enhance the operability of carbon trading laws and regulations, providing the legal basis for the execution of all types of works in China's carbon trading market. Based on the "Measures for the operation and management of clean development mechanism projects" and "interim measures for the management of greenhouse gas voluntary emission reduction", it enacts the measures for the "Management of greenhouse gas compulsory emission reduction", intended to further define the composition parts of carbon trading market and provide the reliable guarantee for the further implementation of carbon market construction works, through the enforcement of compulsory legal force. Finally, in order to truly realize the construction of nationwide carbon trading market, it is necessary to strengthen the development of regional carbon trading market connection mechanism. When the connection mechanism is developed, the relevant laws and systems from other countries may be fully drawn, to ensure the successful achievement of significant functions of connection mechanism.

\subsection{Construction of carbon market legal guarantee system}

The carbon trading administrative body, carbon emission trading platform, carbon pilot enterprises, and third-party verification institution are the indispensable participants of China's carbon trading market. In order to deeply implement the construction works of China's carbon trading market, promote the healthy development of China's carbon market, and ensure that the legal interests of all participants are not violated, government and other relevant departments shall fully play their own roles during the construction process of carbon trading market, and actively enforce the construction works of legal guarantee system, policy guarantee system and market supervision system. The stable operation of carbon trading market cannot be separated from the binding of relevant laws and regulations. In view of the carbon market construction experiences of EU, USA, and other developed countries, China shall improve its existing laws and regulations system. Adopting the legislation as a breakthrough point, it shall definitely point out the fundamental target, operation system, management method and evaluation and measurement methods of carbon trading market operation. Finally, during the construction work of market regulation system, it is necessary to follow the scientific and reasonable basic principles, and fully combine the overall target of national energy conservation and emission reduction, and the regional development conditions and industrial structural features, so as to ensure that the market regulation system will be extensively covered. It is required to set up the special market regulation organization, and define the organizational functions, so as to ensure that the market regulatory functions of organization can be fully realized. Besides, it is also required to set up the rewards and punishment system, to punish the violation acts in the carbon trading market with due severity. As to the legal guarantee, it may choose to independently develop the relevant laws and regulations, but make the perfection on the basis of the existing relevant laws and regulations. 


\section{ANALYSIS OF GLOBAL CARBON TRADING MARKET DEVELOPMENT}

On January 1st, 2018, Ontario province in Canada established the links of its total amount control and trading system with the carbon market of California state in USA, and Quebec province in Canada, forming the third largest carbon market worldwide after China and EU. On December 12th, 2017, Mexico's lower house of Congress (House of Representatives) modified the regulation of "General law on climate change", authorizing the administrative department of the government to establish the nationwide carbon emission right trading system. On November 16th, 2017, Air Pollution Control Board of Virginia Department of Environmental Quality unanimously adopted the draft of "Carbon emission right trading ordinance" in this state. On December 19th, 2017, the South Korean government declared that the total amount control level of Korea Emissions Trading Scheme (K-ETS) in 2018 is set as about 538.5 Million tons, covering almost 600 enterprises. The analysis results of global carbon market development are as follows:

\subsection{Regional carbon market may become the development trend}

The carbon market is the policy market, while its market activity is directly correlated to the policy responses to climate change. The global climate change response target determines the global carbon trading market demands, which is the major push force of carbon trading market development. Since 2020, the climate change response target will directly affect the development future of global carbon trading market. After 2020, it is difficult to form the same compulsory emission reduction framework as the Kyoto Protocol. Instead, it is possible to form the bottom-up loose pattern in which every country will independently determine its own contribution and accept the evaluation. With regard to market mechanism negotiation, each party discusses the Framework for Various Approaches (FVA), multilateral level New Market-based Mechanism (NMM), Non-market Approach (NMA), necessary international and domestic arrangement, applicable statistic framework, verification report and verification methods, and other problems, but still cannot achieve the substantial progress [3].

In this case, the regional carbon market will possibly become the development trend. When the countries where the carbon pricing mechanism is enforced, have the interlinked technical basis and willingness, the interlinked carbon market of a certain scale can effectively mobilize the initiative of market participants, and enhance the market liquidity. In the past year, many countries and regions successively discussed about the possibility of market connectivity, while some have already achieved the substantial progress. It is expected that in some time in the future, such regional carbon market will also appear worldwide.

\subsection{The political game causes that there is still policy uncertainty for some countries to cope with the climate change.}

In recent years, the climate change issue has received more and more attention. Therefore, it also becomes the significant topic for the political parties of major countries to run in election, and hold power. However, due to the political system and decision-making mechanism of developed countries, compared to the national economic and social development and other macro strategies, it is much easier for the climate change policy to have a certain sway. When the EU enforces the "back-loading" plan, it experiences more than one year of "approval-rejection-approval" process which is full of turns and twists. Since the "Market Stabilization Reserve (MSR)" was proposed by the European Commission in 2012, it is still under discussion and negotiation at present, and no final vote has been made. This reflects that the interest relationships inside the EU are very complicated, and there is still some swing for coping with the climate change and economic development related decision making. On July 17th, 2014, Australia officially adopted repeal legislation to abolish the carbon pricing scheme. In its place the government set up the Emission Reduction Fund (ERF) to reduce the emissions, causing the carbon pricing scheme to rush out of the historic stage after being enforced for only two years. The carbon pricing scheme is given up halfway, which is due to the variable emission reduction methods adopted by different political parties of Australia, instead of the institutional problem. Therefore, the enforcement of a good policy requires not only the perfect system design, but also the relatively stable political system and powerful government willingness $[4,5,6]$.

\subsection{The international cooperation is still the main channel and development trend to cope with the climate change in the future.}

The future of carbon trading market still depends on the international cooperation. The diversified international cooperation method will promote the development of carbon pricing scheme, expand the enforcement scope of carbon pricing scheme at the international level, and also form the more extensive policy to respond to the climate change. Firstly, CDM still has the existence value. Under the framework of Kyoto Protocol, CDM has been enforced for many years, which has already been proven to be the effective methods of promoting the emission reduction cost cutting of developed countries, and the sustainable development of developing countries, and promoting the cooperation of response to climate change between developed countries and developing countries. Although the CDM market remains weak at present, it is still necessary to maintain the CDM or similar schemes in the future international agreement regarding coping with the climate change, to continuously promote the cooperation 
of response to climate change between developed countries and developing countries. The cooperation between developed countries and developing countries will welcome its new opportunity. At present, the economic recovery of developed countries is very slow. Under the circumstance that EU ETS and other major carbon market are still weak, the developed countries pay more attention to the carbon trading market of developing countries, hoping to carry out the extensive cooperation. The carbon trading market of many emerging countries or regions is still at the initial stage. Therefore, they are eager to obtain the experiences and technical supports from the developed countries with regard to their system design and market construction. No matter under or beyond the convention framework, such urgency of mutual cooperation will greatly promote the implementation of all types of international cooperation acts to cope with the climate change.

\section{ANALYSIS OF CHINA'S CARBON TRADING COUNTERMEASURES}

The construction of China's carbon trading market system shall adopt the bottom up pattern, namely, to steadily promote in the traditional manner, such as pilot first, promotion second; regional first, nationwide second, spot first, futures second. During the development process from regional pilot to the national market, it is critical for the domestic carbon trading market to solve the problems at two aspects. On one hand, it is critical to create the effective incentive mechanism, so as to develop the enough force to persuade the pilot region to make the trial, and have a certain breakthrough on the compulsory emission market. Simultaneously, it is also required to effectively prevent the region, where the carbon trading pilot is not carried out from reeling in the profits, and fully play the driving role of pilot region, motivating more regions to actively participate in the carbon market. On the other hand, after the regional market of the regional pilot is developed, it is also critical to develop the reasonable mechanism to solve the connection problem between the variable regional markets and nationwide markets. The analysis of China's carbon trading countermeasures is as shown below:

\subsection{It is required to increase the financial support to carbon finance development}

It is necessary to strive to develop the carbon finance, improve the pricing mechanism, and strengthen the publicity and promotion of carbon finance trading. The government shall strengthen its support to carbon finance trading market, increase the publicity and popularization, and allow more financial institutes and non-financial institutions to fully understand the great potential and unlimited business opportunities of the future development of carbon finance in China [7].

6.2 It is required to establish and improve China's laws and regulations regarding carbon

\section{finance trading, providing the legislation protection}

The perfection of laws and regulations is the significant guarantee to fairly and effectively carry out the carbon trade under the market economy conditions, while it is necessary to construct a whole set of laws and regulations system related to the development of nationwide unified carbon market at the earliest. It is necessary to study and develop the relevant laws and regulations to cope with the climate change, such as "Climate change response law or low carbon economy law", collect the carbon tax timely, implement the carbon emission permit system, gradually perfect the policy system and incentive mechanism of climate change mitigation and adaption, modify and perfect the "Envionmenatal protection law, atmospheric pollution prevention and control law", and other special laws at the earliest, and establish the status of market mechanism in carbon emissions reduction. Besides, it is also required to firstly develop the "Measures for the administration of carbon emission permit trading", based on the existing "Measures for the opeartion and management of clean development mechanism projects", and then develop the special "Carbon emission permit trading law", after the carbon trading operation mechanism gradually becomes mature. Therefore, it is especially important for the development of China's carbon trading to establish and perfect the laws and regulations regarding China's carbon finance trading market [8].

\subsection{It is required to instruct and regulate the financial institutions}

It is necessary for the government to develop a complete set of low carbon economy laws and regulations at the earliest as per the domestic carbon finance development conditions, such as tax reduction and exemption of low carbon projects, carbon credit guarantee and investment incentives of low carbon projects, and other policy supports to carbon finance. Meanwhile, the government shall gradually improve China's carbon finance regulation system, such as strengthening the CDM project development and risk management at the delivery link, and enhancing the cross-industry, crossmarket and cross-border carbon finance regulation, etc. Besides, it is necessary to support and guide the financial institutions to carry out the research and practice regarding the forecast, evaluation, prevention and control of carbon finance trading risks. It is necessary for the government and financial institutions to establish the corresponding emergency response plan and emergency response mechanism regarding the carbon finance emergency accidents. Besides, it is also necessary for the government and financial institutions to timely formulate or adjust the relevant business rules, as per the latest progress of carbon finance business, and to establish the up-to-date risk regulation system, risk early alarm system, risk emergency disposal mechanism, and others, so as to make the carbon finance trading business become safer and more reliable [9]. 


\subsection{It is required to strengthen the construction of trading platform, cultivate the carbon trading market system, and energetically cultivate the carbon finance broker service institutions}

It is necessary to establish a fair, open and normalized carbon finance trading market at the earliest, making more and more carbon finance projects realize the transformation from outside trading to inside trading. Besides, it is required to improve the system construction, formulate the matching policies and laws, and actively guide financial institutions and the investors from other organizations to participate in the carbon finance trading. It is also required to cultivate the broking institutions, enhancing the carbon finance service level to a certain level, promoting the effective expansion of scope of participants, and further reducing the cost and risk of project trading $[10,11,12]$.

\subsection{It is required to cultivate the professional carbon finance trading talents, and encourage the diversified design and innovation of carbon finance products}

At present, China's carbon market is still staying at the project level. The lack of professional carbon finance trading talents and inadequacy of relevant products, causes that China has no pricing right on the global carbon finance market. Besides, it also leads to the profit loss of some enterprises and brings a lot of risk pressure to the market. Therefore, it is very important for the construction of China's carbon finance trading system to energetically cultivate the professional carbon finance trading talents, and strengthen the financial supports to the research, development and innovation of carbon finance products [13].

\subsection{It is recommended that Chinese government and financial institutions shall work along both lines}

At the macro level, the government will govern and regulate the development and risk control of carbon finance from the system supply and environmental cultivation perspective. The financial institutions will design the comprehensive and effective carbon finance trading risk pre-alarming index system, construct the perfect carbon finance trading risk management and organization framework. And they will also design and implement the advanced and perfect carbon finance risk management technology, and establish the strict carbon finance trading risk management accountability mechanism. Besides, it is also required to enhance the risk identification and control capacity of carbon finance trading main body and regulation departments.

\section{References}

1. JIANG Mengyue, China's Carbon Trading Development Status, Problems and Countermeasure Analysis: 2015
2. LIU Yameng, TANG Yinhe, LIU Lin, China's Carbon Market Maturity Study: China Market 2015 (13) : 172-174 [3] [3] real time, framework construction carbon market paper, 2015

3. ZHANG Ying, KUANG Jianchao, WANG Zhong, Analysis of Present Development Status of Domestic \& Overseas Carbon Market: Sino-Global Energy, 2014, 19 (3): 1-8

4. LEI Lijun, JIN Zhefeng, Enlightenment of International Carbon Market Development to China: China Population Resources and Environment, 2011, 21 (4): 30-36

5. PENG Juan, XIAO Wei, WEI Qingqi, LEI Xiaoling, Development Situation of Global Carbon Market and Its Enlightenment for Chinese Carbon Market Construction: Environmental Science and Technology, 2013, 26 (5)

6. SONG Haiyun, CAI Tao, Carbon Trading: the Market State, Foreign Experience and Implications for China: Ecological Economy, 2013 (1): 74-77

7. CHU Changxiong, China's Carbon Finance Development Status and strategy: Economist, 2010, 6 (6): $80-86$

8. ZHOU Jian, China's Low Carbon Economy and Carbon Finance Research Overview: Finance \& Economics, 2010 (5)

9. SHENG Xiaojiao, YANG Suchang, Research on Problems \& Strategies Existing in China's Carbon Finance Development, Economic Review, 2011 (11): 89-92

10. YE Yaoming, ZHANG Xifeng, Analysis on Influential Factor of China's Carbon Finance Trading Development [J]: Shanghai Finance, 2010 (7): $31-35$

11. ZHENG Yong, Research on Difficulties \& Countermeasures of Carbon Finance Development in China-Inspirations o $f$ EU Carbon Finance Development: Journal of Xi'an University of Finance and Economics, 2011-9.

12. YANG Yongjie, Mechanism and Present Status of Carbon Trading, China Circulation Economy, 2010 (1):123 -124

13. CHENG Huiqiang, LI Xin, Perfection of Carbon Emission Right Trading Market from Four Aspects: China Venture Capital, 2009 (7): 42-44 